\title{
What can we teach using adaptive optics?
}

\section{Vasudevan Lakshminarayanan}

Vasudevan Lakshminarayanan, "What can we teach using adaptive optics?," Proc. SPIE 9289, 12th Education and Training in Optics and Photonics

Conference, 928906 (17 July 2014); doi: 10.1117/12.2070278

SPIE Event: 12th Education and Training in Optics and Photonics Conference, SPIE. 2013, Porto, Portugal 


\title{
What Can We Teach Using Adaptive Optics? \\ Vasudevan Lakshminarayanan \\ School of Optometry and Vision Science and Departments of Physics and Electrical and Computer Engineering, University of Waterloo, Waterloo, Ontario, N2L 3G1, Canada
}

\begin{abstract}
Adaptive optics (AO) is a powerful technique to sharpen images that are blurred for example due to atmospheric turbulence or aberrations of the eye. Originally developed for defense applications over a period of 40 years or so, they have found enormous applications in the astronomical and vision research communities. This paper provides an overview of adaptive optics techniques. Using principles of $\mathrm{AO}$, it is possible to develop a laboratory that teaches various optics concepts, including aberration theory, at many levels. In addition, AO can teach students some fundamentals of control theory.
\end{abstract}

Keywords: Adaptive Optics, aberrations, control theory, deformable mirrors, Hartmann Shack, wavefronts, visual optics, education

\section{INTRODUCTION}

Adaptive optics is a powerful technique used to sharpen images blurred, for example by atmospheric turbulence or aberrations of the eye. Originally developed for defence applications, the methods were introduced to the astronomical community for use in ground based telescopes in the late 1980s. In fact with adaptive optics techniques it is possible to produce images in ground based telescopes with quality rivaling that of the Hubble telescope or other space based telescopes currently being planned. The history of the development of AO is covered for example, in the book by one of the pioneers in the field, Robert Duffner ${ }^{1}$. The principles of adaptive optics is dealt with in the classic monograph by Tyson ${ }^{2}$. Tyson has also written a rather humorous account of $\mathrm{AO}^{3}$. The reader can refer to the book by Roddier ${ }^{4}$ or the recent review by Davies and Kasper ${ }^{5}$ for a glimpse into astronomical applications. AO was adapted for use in studying the aberrations of the human eye by Liang et $\mathrm{al}^{6}$. A good review of vision science applications can be found in the article by Hampson ${ }^{7}$ and the books by Porter et $\mathrm{al}^{8}$ and Dai ${ }^{9}$. In addition to these two areas adaptive optics techniques are also used in manufacturing (photolithography for example), lasers, communications and in biomedical optics ${ }^{10}$. The reader is also urged to look at the website of the Center for Adaptive Optics located at the University of California at Santa Cruz (http://cfao.ucolick.org/).

\section{ADAPTIVE OPTICS: BASICS}

Why do we need adaptive optics at all? This is because of optical aberrations present in any optical system. Aberrations are the inherent shortcomings of an optical system. Within the framework of geometric optics, a perfect optical system is stigmatic (latin for point-like) that is, each object point focuses to a single image point. Wavefronts emerging from a point object are always spherical (divergent), and after being transformed by the stigmatic optical system, converging wavefronts are again spherical, but now centered around the image point. An object point at optical infinity produces a planar wavefront. An aberration is any departure from sphericity/planarity or shift in centration of a converging wavefront leading to blurred or systematically moved image points. There are two types of aberrations: chromatic aberration and monochromatic aberrations. Aberrations can either deteriorate the image, or deform the image (or of course both). AO deals with correction of monochromatic wavefront aberrations.

*vengu@uwaterloo.ca; phone:1.519.888-4567ext.38167; fax:1.519.725.0784

12th Education and Training in Optics and Photonics Conference, edited by

Manuel F. P. C. Martins Costa, Mourad Zghal, Proc. of SPIE Vol. 9289, 928906

(c) 2014 SPIE, OSA, IEEE, ICO · doi: 10.1117/12.2070278

Proc. of SPIE Vol. 9289 928906-1 
$\mathrm{AO}$ is a scientific and engineering discipline wherein the performance of an optical signal is improved by using information about the environment in which it passes. The optical signal can be light from a star or a laser beam passing through the air. Here the aberrations are dynamic due to the random Markovian nature of atmospheric currents. It is requirement to propagate/receive an undistorted beam that has developed the field of $\mathrm{AO}$. AO can also be described as a method of automatically keeping light focused when it is out of focus. AO deals with the control of light/optical system in a real-time closed loop fashion. Therefore, AO is a sub-set of active optics. AO and active optics are differentiated mostly by the bandwidth in which they function. Active optics systems work below $1 / 10 \mathrm{~Hz}$ while AO works in the greater than $1 / 10 \mathrm{~Hz}$ regime. All AO systems basically consist of a wavefront/phase sensor (such as a Hartmann Shack device), an active wavefront shaping element (such as a deformable mirror) and a controller that connects the sensor and the active device as well as feedback to monitor image quality. The basic outline of an AO system is shown in Figure 1 .
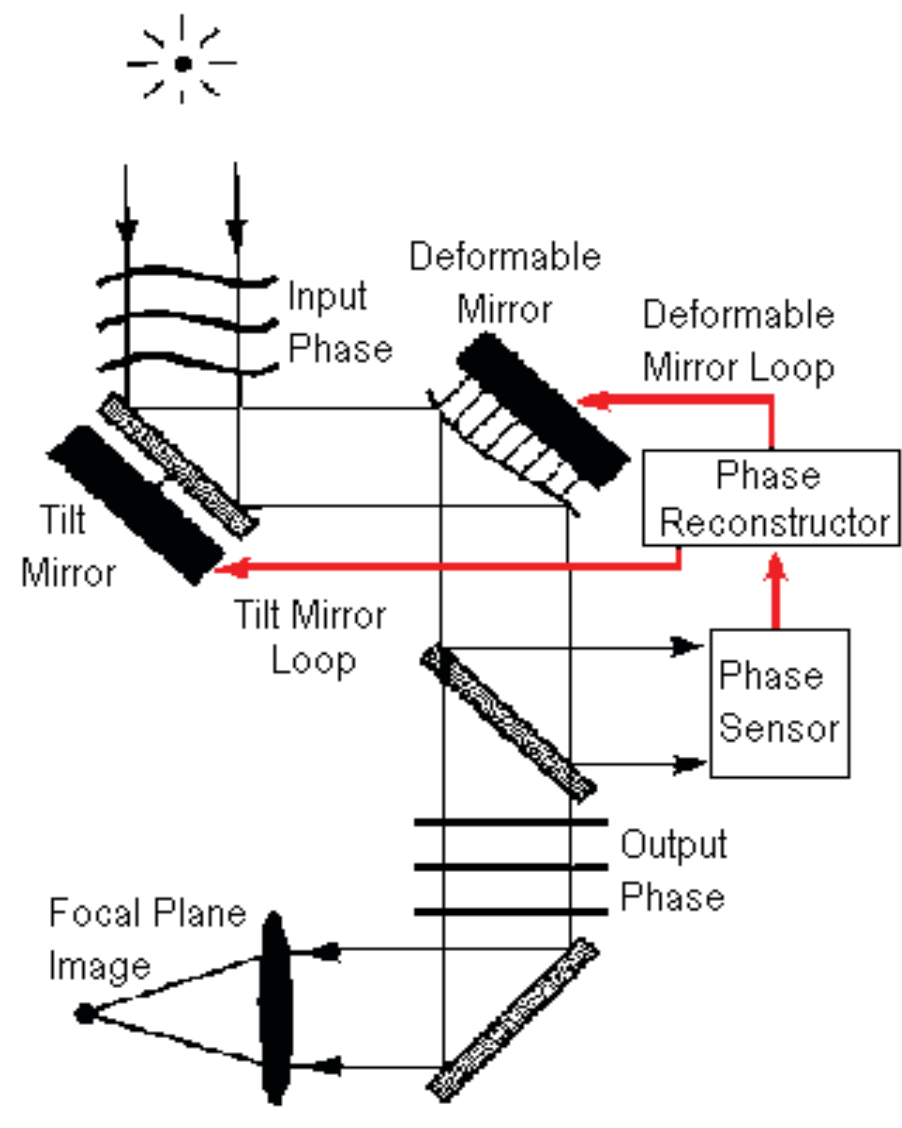

Figure 1:This simplified view of the adaptive optics system for the Gemini 8-m Telescopes Project. A series of tiltmirror and deformable mirror corrections are applied to compensate as much as possible for the blurring and distorting effects of the atmosphere on the light from the source. From : The Gemini 8-m Telescope project, www.gemini.edu/science.

The principles of $\mathrm{AO}$ are based on the premise that one can change the imaging effects of an optical system by adding, altering or removing optical elements. With AO it is not possible to get rid of diffraction effects, but is possible to reach diffraction limits. In all AO systems, the wavefront incident on the system is the quantity to be changed to alter the propagation characteristics of the beam. It is a two dimensional of the phase at an aperture (usually the entrance pupil of 
the system) which is normal to the line of sight between the origin and the target. In general, the two dimensional wavefront map can be represented by a power series in polar co-ordinates and hence, a Taylor polynomial. However, since the Taylor series is not orthonomalized, customarily, a special orthonormal polynomial set, called Zernike polynomials are used as the basis function to express the wave aberration. The Zernike series is composed of sums of power series terms with appropriate normalization factors. Because of the orthogonal nature, addition of more terms does not change the lower order terms. This method of representation is also attractive since it is orthonormal over the entire cicular pupil. Zernike polynomials are a form of hypergeometric functions and details can be found in the paper by Lakshminarayanan and Fleck ${ }^{11}$.

AO systems in general incorporate feedback loops and are control systems. If feedback is employed it is called a closed loop and applies to both positive and negative feedback. In an open loop system, there is no feeback. If the optical information is gathered at the image plane it is called a target loop and if the information is gathered before the image plane, the short-circuited loop is called a local loop. A good example of an AO system in a closed loop feedback is the eye. The eye is capable of adapting to various conditions to improve the image quality on the retina. The active focus (accommodation) of the visual system is a perfect example of AO. The brain interprets the retinal image, determines the correction required and then applies the necessary correction through the biomechanical movement of the crystalline lens. Here the eye uses feedback to correct the image, through both eye movements as well as changes in the shape of the crystalline lens of the eye and is a good example of a feedback control system ${ }^{12}$. The eye can also follow the movement of an object. This is a form of a tilt mode AO system (where there is a variation in the beam direction or tilt of the wavefront). Lastly, the pupil changes shape in response to light levels (again a feedback control system) ${ }^{13}$. This is a demonstration of $\mathrm{AO}$ in an intensity control mode.

The wavefront sampling subsystem redirects a portion of the incoming wave to the wavefront sensor. This is usually accomplished with a high quality beam splitter. The wavefront sensor should get a sample of the wavefront that is a high fidelity reproduction of the wavefront that needs correction.

There are two types of wavefront sensing, one, the direct approach and two the indirect approach. In the direct approach there is a determination of phase. After the wavefront is reconstructed, the information is used as feedback to correct for unwanted components of the phase. In the indirect approach, information related to phase is translated into signals that are used to compensate for the wavefront. In modal sensing, the wavefront is expressed in terms of coefficients of the modes of the polynomial expression over the entire pupil, whereas in zonal sensing the wavefront is expressed in terms of optical path difference over a small spatial area. In zonal direct wave sensing, the reconstruction of the wavefront of the entire field is made from the measurement of tilt in smaller regions. This method applies to all orders of aberrations. Sensing the focus of a beam directly is fundamental to any modal AO operation. The focus aberration is the most heavily weighted aberration when it comes to smearing of the image. Interferometric wave sensing can also be used to generate the contours of the wavefront, as well as the local wavefront tilt.

One of the most popular methods of measuring wavefronts is the use of the Hartmann Shack wavefront sensor. Historically, testing lenses or mirrors employed an opaque mask with holed placed behind the optical element under test. Each hole would act as an aperture and since passes through the lens is converging an array of spots will be produced. With proper calibration, the position of the spot is a direct indication of the local wavefront tilt at each hole and thus a description of lens quality. This is called the Hartmann tes ${ }^{14}$. Variations of this method are used in the AO wavfront sensors. Ronald Shack (and B.C.Platt)placed lenses in the holes, which increased the light gathering efficiency of the mask and reduced the effects of diffraction caused by the holes. ${ }^{15}$ These lenses produce a spot of light $\mathrm{n}$ a CCD array.

A second type of sensor is the curvature sensor ${ }^{16}$. Curvature sensing measures local wavefront curvatures in each aperture. By comparing the two near simultaneous irradiance distribution at equally spaced points of either side of a sub-aperture focal plane the local curvature can be found. The advantage of curvature sensors is that they are often less costly than HS sensors and have a higher dynamic range. The pyramidal sensor is another method to measure the wavefront. The pyramidal sensor is in principle similar to the Foucault knife edge test. Here, a pyramid of transparent material is placed such that the apex of the pyramid is at the focus of the beam whose wavefront variation over the aperture is needed. The converging rays are split into four images which are detected on different parts of a CCD camera. The variation of intensity in each quadrant will be measured and the wavefront reconstructed. Lensless and digital reconstruction methods have also been developed recently which have greater efficiency. These include replacing the lenslet array in a HS sensor with a two dimensional grating or a spatial light modulator. ${ }^{17,18}$ More recently sensorless imaging techniques have been used especially in microscopy. The basic principle is that if optical aberrations affect the quality of the image then some information about the nature of the aberration must be encoded in the image. Therefore, 
some of this information can be extracted from the image. This is an example of indirect wavefront sensing. ${ }^{19}$ The visual system is an example of the indirect wavefront sensor.

Image sharpening ${ }^{20}$ is a technique that is sometimes used. The intensity in the focal plane must be such that the image degraded by some disturbance can be recovered. This requires manipulation of the phase correcting elements while observing some function of the image. This technique is used for the correction of degraded images in cases where sharp images are required, and hence the term image sharpening. This technique requires the maximization of a function defined as sharpness. By changing the phase of various parts of the imaging system the intensity at one point (usually the center) can be maximized. The most common image sharpness that is maximized is the integral of the square of the image intensity distribution.

The wavefront correction can be achieved by many of the same mechanisms that cause wavefront distortions. For example, an image from a distorted mirror can be corrected by distorting another mirror into proper shape to compensate for the wavefront variations. In dynamic AO, this is achieved by distorting the mirror (or optics) that receives the aberrated wavefront. Local variations in refractive index that were the cause of aberratins can be reversed or conjugated to provide correction. In the human eye for example, we use corrective lenses - spectacles, contact lenses. The simplest form of wavefront correction is variation of the beam direction or the tilt of the wavefront. Segmented mirrors are made up of a number of closely spaced small mirrors with piston or tilt correction capabilities.

Deformable mirrors are continuous suface mirrors with mechanical means of deformation to match the desired conjugate wavefront ${ }^{21}$. This is achieved by using actuators. These can be either continuous as in membrane or bimorph mirrors or discrete. The actuators can be perpendicular to the surface or on the edge imparting bending movements. By moving the actuators in the same plane as the surface, bending movements can be induced that correspond to focus, etc. A bimorph mirror which is deformable consists of a glass or metal mirror faceplate bonded to a sheet of piezoelectric ceramic, which is polarized normal to its surface. The reader is referred to the literature for further details. A more recent development is the use of liquid crystal spatial light modulators, which one can manipulate by changing the orientation of the liquid crystals by using electrostatic forces. This will vary the refractive index of the material spatially and therefore the phase and/or amplitude of the wavefront can be changed ${ }^{22}$.

The feedback control has a number of technical questions also. The controller develops the appropriate voltages, for example, for the actuators to modify the deformable mirrors. For example, what is the most optimal controller in such systems with feedback? Many models use a linear-quadratic-Gaussian system for the control model. There are a number of highly sophisticated mathematical algorithms to answer this question. A good review of some of these based on linear control theory is given in a recent article by Folcher et $\mathrm{al}^{23}$.

Given all these factors what does a real life AO system look like? The answer is simple - many of the giant modern telescopes around the world use some form of AO. These include, the Subaru (National astronomical Observatory of Japan), Gemini (Hawaii and Chile),CFHT (Hawaii),Mt.Wilson (California),Palomar(California),Keck(Hawaii) and VLT(Chile). I will conclude this section with pictures of AO assembly in my laboratory, a compact system for measuring the wavefront aberrations of the human eye.

Figure 2: HS AO system to study aberrations of human eye; left: side view; right: top view

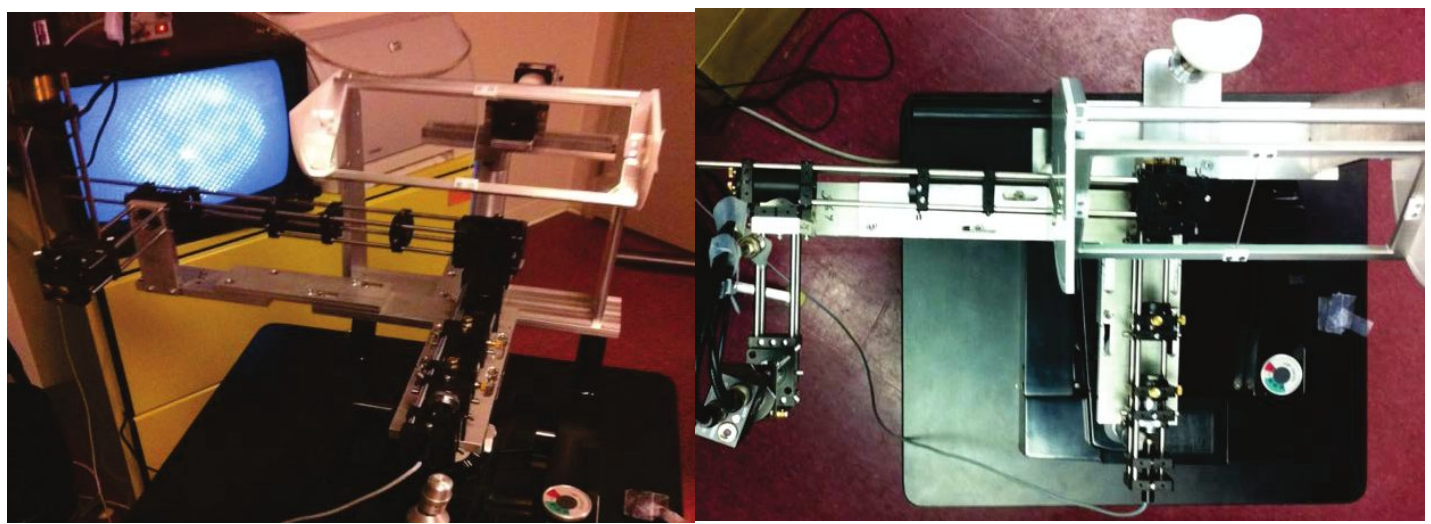




\section{TEACHING WITH ADAPTIVE OPTICS}

It should be noted that in most physics courses (both in high school and in freshman college) optics is not given its rightful place. Typically the student is exposed to some geometrical optics and image formation by lenses, etc. along with a chapter on interference, diffraction and polarization. Rarely is the student exposed to more advanced topics in optics. Optics, is the "enabling science" as described by the National Research Council in its influential report on optical sciences $^{24}$. AO is rarely discussed even in college classes on physics (though some astronomy introductory courses do discuss $\mathrm{AO}$ ) due to its complexity, but has the potential to be of great use in optics education.

As noted earlier, $\mathrm{AO}$ can be used to teach a number of different concepts in optics at various grade levels. In addition, it is possible to teach, for example, aspects of visual optics and the human visual system. The Center for Adaptive Optics has developed AO educational materials for college students. However, these materials are expensive(deformable mirrors, HS sensors, etc.) and cannot be used for example in high schools.

What exactly can one learn conceptually from AO? The first lesson is that light is actually a wave and the concept of wavefront is put forth as being primary. The next concept to be learnt is that these wavefronts can be distorted due to atmosphere or by optical systems. These distortions are nothing but aberrations. The student can also learn that if there is an aberrated system, the image is no longer perfect (i.e., is distorted) - this can be easily demonstrated by having a mirror (or a piece of highly metallic film) and using a ray box to show that these rays no longer come to a focus. The concept of deformable mirrors can be used to show how flexing the mirrors can easily overcome the distortions. This for example can be done in an interactive lecture demonstration set-up.

In a previous paper I have argued that the human eye can be a perfect system for teaching optics ${ }^{25}$. This is even more applicable with AO systems. I have already given examples (vide supra) of this here. In my courses on visual optics, I use these ideas to reinforce basic understanding of Optics. The idea of feedback is fundamental in nature and can be found in biological, chemical and physical systems. AO offers the possibility to teach ideas of feedback and control which are rarely taught in introductory courses.

Steve Pompea and his group at the National Optical Astronomical Observatory have developed kits for adaptive optics teaching in high school ${ }^{26}$. These kits which utilize hands-on activities build a conceptual framework for understanding image formation, mirrors and AO, using very little mathematics. This is a much needed, welcome addition to the handson, minds-on active learning mode of teaching.

There is also a need for simulations - with the advent of easy to use software such as Mathematica or Matlab, it is easy to simulate various aspects of optics in general and $\mathrm{AO}$ in particular. Here, it has been my experience that a good GUI (graphical user interface) is always required and as the usual saying goes , a picture is worth a thousand words. Hence, the results of the simulation has to be displayed in a easily understandable, eye-catching way. With more advanced students, it is possible that using a simple language like Python, it is possible to have them program various situations within a short time period. At the University of Michigan freshman students in physics learn within a few lectures to program interesting problems in Perl or Python (Prof. Brad Orr, personal communication, June 2013). The key here is to not be lost amongst the trees (the actual mechanics of programming and computation) and miss sight of the forest (the physics).

\section{ACKNOWLEDGEMENTS}

I would like to thank Dr. Steve Pompea for providing me a version of the adaptive optics kit developed by him and his group at NOAO, Tucson, AZ. The author is also affiliated with the Department of Physics at the University of Michigan for the year 2013-2014 and the Michigan Center for Theoretical Physics at UM. 


\section{REFERENCES}

[1] Duffner, R.W. The Adaptive Optics Revolution, A History; University of New Mexico Press: Albuquerque, NM, (2009).

[2] Tyson, R.K. Principles of Adaptive Optics, 3rd ed.; CRC Press: Boca Raton, FL.(2011).

[3] Tyson, R.K., The Lighter Side of Adaptive Optics, SPIE, Bellingham, WA. (2009)

[4] Roddier, F. Adaptive Optics in Astronomy; Cambridge University Press: Cambridge, UK (1999)

[5] Davies, R., and Kaspur M., "Adaptive optics for astronomy", Ann Rev Astron Astrophysicics, 50:305-351,(2012)

[6] Hampson, K. "Adaptive Optics and Vision", J. Mod. Opt. 55:3425-3467 (2008)

[7] Porter, J.; Queener, H.; Lin, J.; Thorn, K.; Awwal, A. Adaptive Optics for Vision Science; Wiley, NY.(2006)

[8] Dai, G. Wavefront Optics for Vision Correction; SPIE Press:Bellingham, WA.(2008)

[9] Liang, J.,Grimm, B.,Goelz, S. and Bille J.F.," Objective measurement of wave aberrations of the human eye with the use of a Hartmann-Shack wave-front sensor" J. Opt.Soc. Am. A., 11(7):1949-1957(1994)

[10] Kubby, J.A., Adaptive Optics for Biological Imaging, CRC Press: Boca Raton, FL. (2013).

[11] Lakshminarayanan, V.,Fleck, A. J. Mod. Opt. 58:545-561,2012.

[12]V.Srinivasan, W.Bobier, E.Irving, V. Lakshminarayanan, Vergence adaptation to accommodative vergence: comparison of model simulations with experimental data, IEEE Trans. Biomed. Engineering, 56:2389-2395, (2009).

[13] Stark, L. Neurological Control Systems, Plenum Press:NY(1968)

[14] Hartmann J., “Objektuvuntersuchungen,” Zt. Instrumentenkd., 24, 1 (1904)

[15] Shack R.V., and Platt, B.C., J. Opt. Soc. Am., 61:656, abstract only, (1961).

[16] Hickson, P., and Burley, G.S. "Single image wavefront curvature sensing", Proc. SPIE, 2201, Adaptive optics in astronomy, 549, doi:10.1117/12.176090, (1994).

[17] Zhao, L., Bai,N., Li,X., et al., "Efficient implementation of a spatial light modulator as a diffractive optical microlens array in a digital Shack-Hartmann wavefront sensor", Appl. Optics, 45(1):90-94,(2006).

[18] Lai, S.C., King, B., and Neifeld, M.A.,"Wavefront reconstruction by means of phase shifting digital in-line holography", Opt. Commun., 173:155-160(2000)

[19] Booth, M.J., "Wavefront sensorless adaptive optics for large aberrations", Opt. Letters, 32(1):5-7,(2007)

[20] Muller, R.A., and Buffington A. "Real-time correction of atmospherically degraded telescope images through image sharpening", J. Opt. Soc. Am., 64(9):1200-1210, (1974).

[21] Babcock, H.W., "The possibility of compensating astronomical imaging”, Publ.Astron.Soc.Pac., 65:229-236 (1953).

[22] Milkie, D.E., Betzig, E. and Ji.N. "Pupil-segmentation based adaptive optics with full pupil illumination", Opt. Letters, 36:4206-4208 (2011).

[23] Folcher,J-P, Carbillet, M., Ferrari, A., and A. Abelli., “Adaptive Optics Feedback Control”, EAS Publications Series, 59: 93-130. doi:10.1051/eas/1359006 (2013).

[24] National Research Council,"Harnessing Light”, National Academies Press:Washington DC,(1998).

[25] Lakshminarayanan, ., "The human eye as a model system for teaching optics", in Education and Training in Optics and Photonics, OSA Technical Digest series, Paper ESCB5, www.opticsinfobase.org/abstract.cfm?URL=ETOP2009-ESCB5, (2009) .

[26] Sparks,R.T.,, Pompea,S.M,, WalkerC.E.,, Doktor,E.F.”Teaching Adaptive Optics Concepts in the High School Classroom Using an Active Engagement, Experimental Approach" Proc. SPIE, 7783, doi: 10.1117/12.862633, (2010) 